ARTICLE

DOI: $10.1038 / s 41467-017-02428-w$

\title{
Punctuated evolution of canonical genomic aberrations in uveal melanoma
}

\author{
Matthew G. Field ${ }^{1}$, Michael A. Durante ${ }^{1}$, Hima Anbunathan², Louis Z. Cai ${ }^{1}$, Christina L. Decatur ${ }^{1}$,
} Anne M. Bowcock (D) ${ }^{2}$, Stefan Kurtenbach ${ }^{1} \&$ J. William Harbour (i) ${ }^{1}$

Cancer is thought to arise through the accumulation of genomic aberrations evolving under Darwinian selection. However, it remains unclear when the aberrations associated with metastasis emerge during tumor evolution. Uveal melanoma (UM) is the most common primary eye cancer and frequently leads to metastatic death, which is strongly linked to BAP1 mutations. Accordingly, UM is ideally suited for studying the clonal evolution of metastatic competence. Here we analyze sequencing data from 151 primary UM samples using a customized bioinformatic pipeline, to improve detection of BAP1 mutations and infer the clonal relationships among genomic aberrations. Strikingly, we find BAP1 mutations and other canonical genomic aberrations usually arise in an early punctuated burst, followed by neutral evolution extending to the time of clinical detection. This implies that the metastatic proclivity of UM is "set in stone" early in tumor evolution and may explain why advances in primary treatment have not improved survival.

\footnotetext{
${ }^{1}$ Bascom Palmer Eye Institute, Sylvester Comprehensive Cancer Center and Interdisciplinary Stem Cell Institute, University of Miami Miller School of Medicine, Miami, Florida 33136, USA. ${ }^{2}$ National Heart and Lung Institute, Imperial College London, London, SW3 6LR, UK. Correspondence and requests for materials should be addressed to J.W.H. (email: harbour@miami.edu)
} 
U veal melanoma (UM) is the most common cancer of the eye and leads to metastatic death in up to half of patients. UMs are commonly divided into two prognostic subgroups based on their gene expression profile (GEP); those with the class 1 GEP have a low risk of metastasis, whereas those with the class 2 GEP have a high risk ${ }^{1}$. UM is also notable for having two sets of driver mutations, with each tumor typically containing one mutation from each group ${ }^{2}$. One group consists of mutually exclusive gain-of-function mutations in members of the Gq signaling pathway-GNAQ, GNA11, CYSLTR2, or PLCB4. These "Gq mutations" are present in virtually all UMs, are not prognostically significant, are insufficient alone to induce malignant transformation, but are seemingly required to initiate tumorigenesis $^{3-8}$. The other group consists of near-mutually exclusive mutations in BAP1, SF3B1, and EIF1AX ("BSE" mutations), which are strongly prognostic of metastatic risk. Inactivating mutations in the tumor suppressor BAP1 are associated with the class 2 GEP and high metastatic risk ${ }^{9}$, whereas single nucleotide substitutions in SF3B1 and EIF1AX are found mainly in class 1 tumors and are associated with intermediate and low metastatic risk, respectively ${ }^{10,11}$. UMs are also associated with a small set of recurrent chromosome copy number alterations (CNAs), which tend to occur in the context of a specific GEP class and BSE mutation ${ }^{12}$. Loss of heterozygosity for chromosome 3 (LOH3) is frequently found in BAP1-mutant class 2 tumors and is thought to represent the "second hit" in the bi-allelic loss of BAP1, located at chromosome $3 \mathrm{p} 21^{9}$. $6 \mathrm{p}$ Gain $(6 \mathrm{p}+)$ is often found in class 1 tumors harboring SF3B1 and EIF1AX mutations, whereas $8 \mathrm{q}$ gain $(8 \mathrm{q}+)$ can be found in both class 1 and class 2 tumors, and is associated with $B A P 1$ and SF3B1 mutations.

Despite success in identifying these canonical genomic aberrations in UM, how and when these events arise during tumor evolution remains unknown. In cutaneous melanoma, progression from benign nevus to malignant melanoma follows a typical Darwinian model of gradual evolution ${ }^{13}$, characterized by successive waves of mutations, clonal expansions, and selective sweeps fueled by ultraviolet radiation-induced DNA damage, with CNAs occurring relatively late ${ }^{14,15}$. An obstacle to performing reliable genomic clonality analysis in UM has been an inability to detect the wide diversity of BAP1 mutations using standard bioinformatic methods. Here we analyze next generation sequencing (NGS) data from 151 primary UMs using a wide range of bioinformatic tools and techniques to optimize our detection of BAP1 and other mutations and CNAs, to explore their clonal relationships. This approach reveals many previously undetected BAP1 and spliceosome mutations, and uncovers strong evidence that the canonical genomic aberrations in UM usually arise in an early, punctuated burst followed by clonal stasis. These findings underscore the striking differences in genomic structure and evolution between UM and cutaneous melanoma, and they have profound implications for treatment and survival in UM.

\section{Results}

Data sets for genomic analysis. We initially analyzed whole exome sequencing (WES) data from 139 primary UM samples, including 37 from the practice of the senior author (J.W.H.), 80 from the The Cancer Genome Atlas (TCGA), and 22 from a publicly available data set (UNI-UDE) ${ }^{11}$ to identify driver mutations and CNAs (Fig. 1, Supplementary Table 1, and Supplementary Data 1). GEP classification data were available for all JWH samples and was estimated using RNA sequencing (RNAseq) data for TCGA samples ${ }^{16}$.

Detection of BAP1 mutations. Recent analyses have found $B A P 1$ mutations in only about $20 \%$ of $\mathrm{UMs}^{11,17}$, yet $\sim 40 \%$ of UMs are expected to harbor these mutations ${ }^{9}$. Thus, we suspected that BAP1 mutations may frequently be missed when they comprise large insertions/deletions (indels), intronic/splicing alterations, and other complex rearrangements of the BAP1 locus ${ }^{9}$. To enhance our ability to detect such a wide range of inactivating mutations, we developed a robust pipeline of complementary bioinformatic tools to improve read alignments, manage low read counts, and identify large genomic alterations (Supplementary Fig. 1). In comparison with the Firehose analysis ${ }^{17}$, which detected BAP1 mutations in 17 (21\%) of the 80 TCGA samples using the GATK pipeline (Supplementary Fig. 2), our pipeline detected BAP1 mutations in $36 / 80(45 \%)$ of the same samples (Supplementary Data 1). Similarly, Martin et al. ${ }^{11}$ identified BAP1 mutations in $4(18 \%)$ of 22 samples, whereas we identified $B A P 1$ mutations in $9(41 \%)$ of the same samples (Supplementary Data 1). Overall, BAP1 mutations were detected in $62(45 \%)$ of

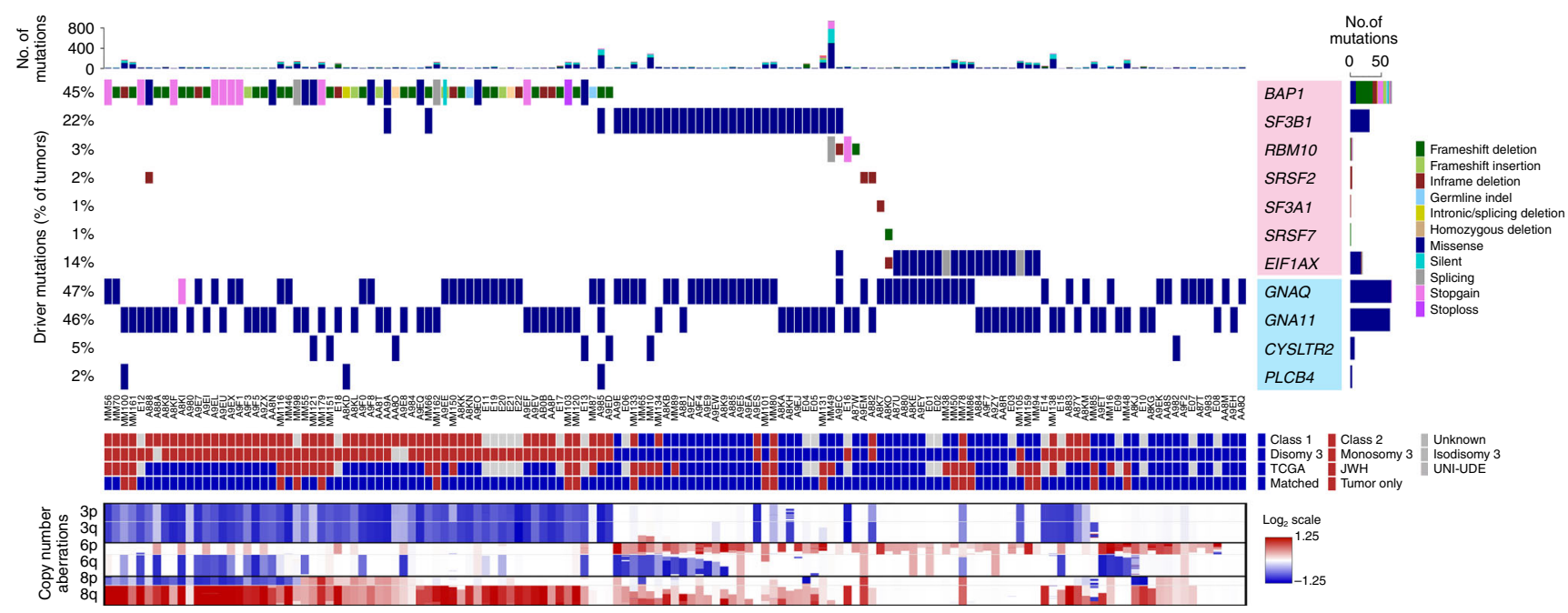

Fig. 1 Molecular landscape in 139 primary uveal melanomas analyzed by whole exome sequencing. Mutation status for common driver and spliceosome mutations, type of mutation, common chromosome copy number alterations (CNAs), gene expression profile status (class 1 versus class 2), source of tumor sample, and availability of matched normal DNA are indicated. CNAs were assessed using CNVkit ${ }^{62}$. CNA data are scaled using the log2 copy ratio of the predicted copy number over the normal copy number. Mutations in "BSE" and splicing genes (pink box) are demarcated from those in Gq signaling pathway genes (blue box) 
SNP

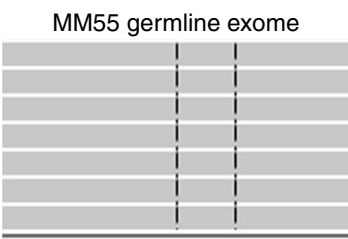

MM55 tumor exome

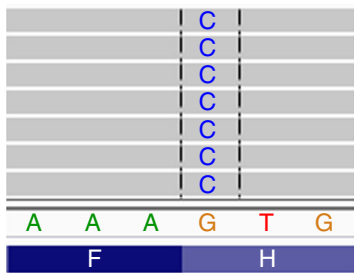

RNA-seq rescue

A8KF tumor exome

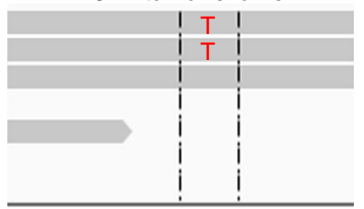

A8KF tumor RNA-Seq

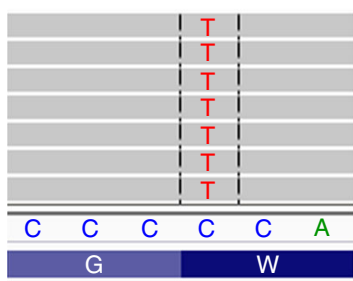

Small indel

MM70 germline exome

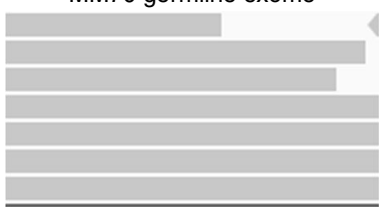

MM70 tumor exome

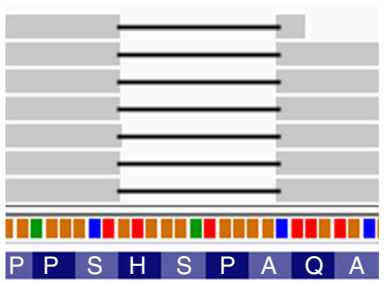

RNA-seq "SpliceDel" A9F5 tumor exome

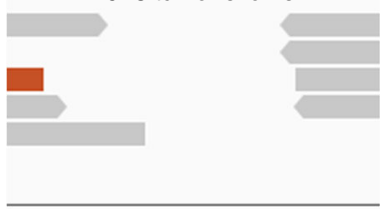

A9F5 tumor RNA-Seq (STAR)

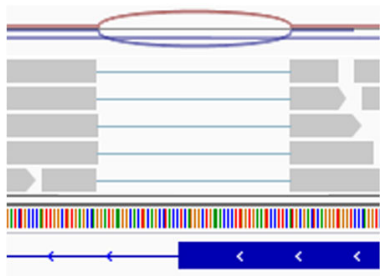

Large indel

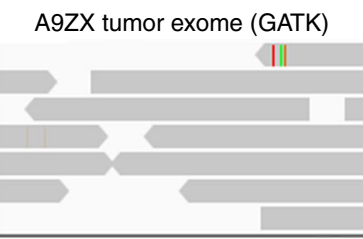

A9ZX tumor exome (ABRA)

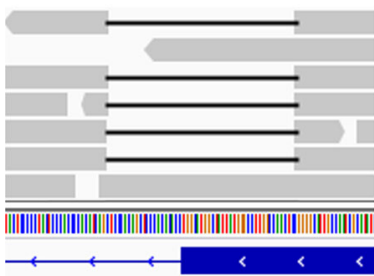

WGS "SpliceDel" T4 tumor WGS

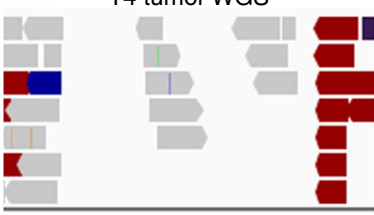

T4 tumor WGS (STAR)

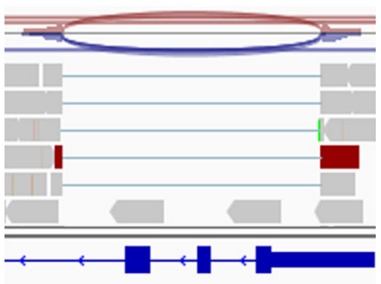

Germline mutation

MM87 germline exome

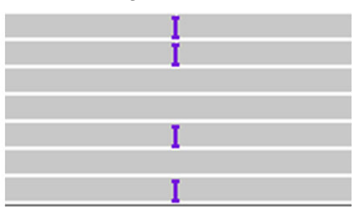

MM87 tumor exome

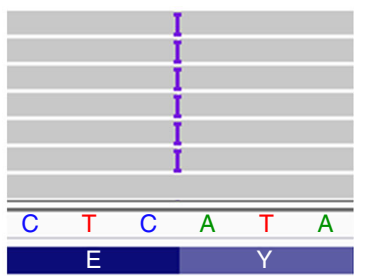

Homozygous deletion

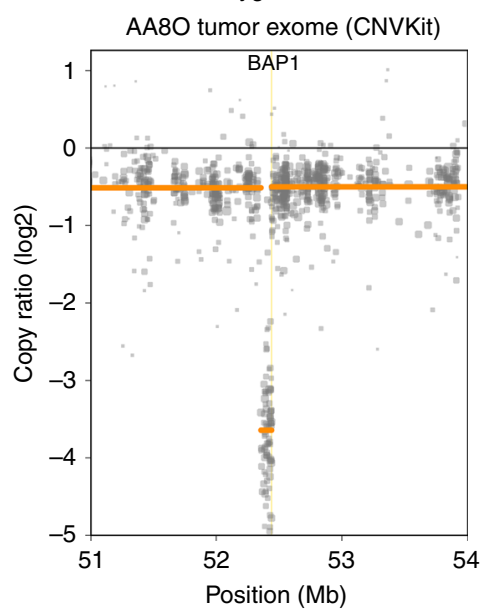

Fig. 2 BAP1 mutation diversity and necessary detection methods. Standard somatic mutation callers (i.e., Varscan2 and MuTect2) can detect SNPs (SNP) and small indels (small indel). Large indels need specialized realignment (i.e., with ABRA) for detection, as these can be missed with IndelRealigner and/or Mutect2 (large indel). Somatic mutation callers exclude germline mutations, so blood samples need to be run with a germline mutation caller (e.g., HaplotypeCaller) or as an unmatched "tumor" with a somatic caller (e.g., MuTect2) to detect patients with BAP1 hereditary cancer predisposition syndrome (germline mutation). Mutations can be "rescued" in low coverage regions by combining WES and RNA-Seq data (i.e., UNCeqR) (RNA-seq rescue). Large indels may be missed with WES data when they start or extend into intronic or promoter regions due to poor bait coverage. In the case of the former, RNA-Seq data can be used to detect large indels that start in a transcribed exon and extend into the intron using alternative splicing algorithms (RNA-seq "SpliceDel"). For the latter, WGS with alternative splicing algorithms is required to detect indels that start in the promoter region or 5'-UTR and extends into exons or across multiple exons (WGS "SpliceDel"). In cases with loss of one chromosome 3 and deletion of genes on the remaining copy of chromosome 3, a CNA caller is required to detect the homozygously deleted regions (homozygous deletion). Sample alignments were visualized using Integrative Genomics Viewer. A representative sample was selected for each mutation type and detection method

the 139 WES samples queried in this study, including 9 nonsynonymous single-nucleotide polymorphisms (SNPs), 10 stopgain/stoploss SNPs, 2 intronic/splice SNPs, and 41 indels (Fig. 2 and Supplementary Data 1). The use of two different mutation callers (MuTect2 and Varscan2) improved detection of BAP1 mutations, 17 of which were only called by one algorithm or the other. Using ABRA to re-align around large indels ${ }^{18}$, we detected $B A P 1$ deletions of $>20$ base pairs in 10 samples that were not previously detected by other methods (Fig. 2). In one sample (A8KF), we detected a BAP1 mutation that was initially filtered out due to low exome coverage but was "rescued" with matched RNA-Seq data using UNCeqR ${ }^{19}$ (Fig. 2). In addition, due to the design of baits for exome capture, large deletions that start or end in intronic or promoter regions can be missed using WES data. In three samples (AA8P, A9F5, and AB0B), we used RNA-seq data and the STAR aligner to identify large splicing deletions ("SpliceDels") that start in an exon and extend into an intron (Fig. 2).
These samples had low DNA coverage in the deleted regions, yet all three showed at least one read that called the deletion and multiple reads on either side of the deletion that did not span into the deleted region, corresponding precisely to the "SpliceDel" mutations detected by RNA-seq. In two samples (E21 and AA8O), we identified homozygous deletions of the BAP1 locus using CNVkit that would be missed by most mutation-calling algorithms (Fig. 2). In two samples that were initially filtered out by our pipeline due to low coverage (A9EQ and E22), BAP1 mutations were identified by visual inspection using the Integrative Genomics Viewer (IGV). A9EQ showed the same mutation in both WES and RNA-seq data, and E22 showed an obvious deletion in three out of six reads. Somatic mutation callers pair germline and tumor samples to filter out germline SNPs. However, as BAP1 mutations are known to occur in the germline in some cases, we analyzed all blood samples independently of their matched tumor using MuTect2, with which we identified two 


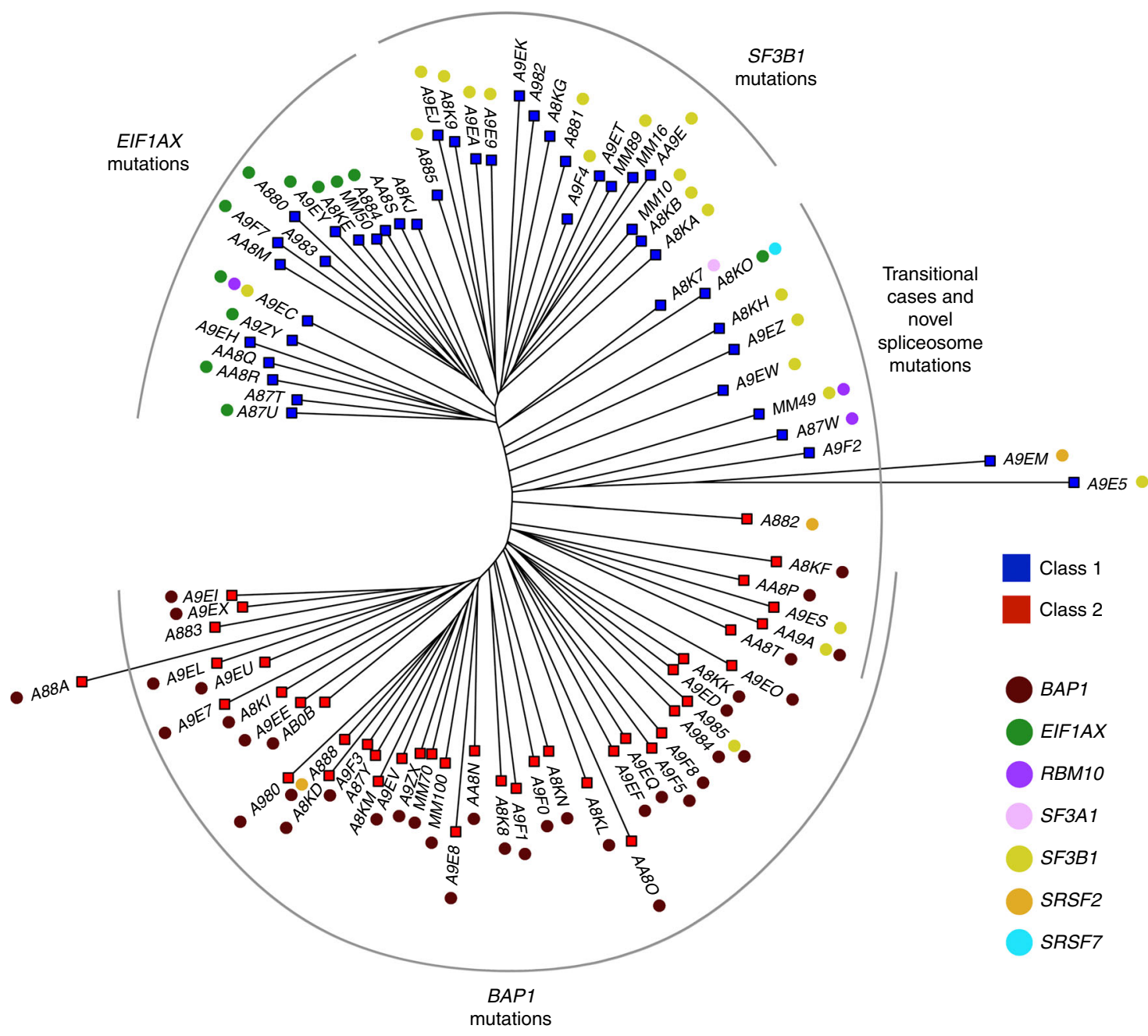

Fig. 3 Phylogenetic tree demonstrating relationships between uveal melanoma samples based on genomic DNA methylation. A minimum evolution algorithm (Canberra distance) was used to build an unrooted evolutionary tree. Gene expression profile (class 1 versus class 2 ) and mutation status are indicated. These data are presented in a rooted phylogenetic tree with significance estimates in Supplementary Fig. 5

cases with germline BAP1 mutations (MM87 and A8KN) (Fig. 2). Even with our optimized pipeline, we did not identify a BAP1 mutation in 10 of the class 2 samples; thus, we suspected that some BAP1 mutations are not detectable with WES or RNA-seq data, because they span multiple exons or start in untranscribed regions. Indeed, when we analyzed publicly available whole genome sequencing (WGS) data from $12 \mathrm{UM}$ samples reported by Furney et al. ${ }^{20}$ using our "SpliceDel" technique, we identified 2 samples containing multi-exonic BAP1 deletions that started in the $5^{\prime}$-untranslated region and intronic regions (Fig. 2 and Supplementary Data 2), which were previously unreported. Hence, a complimentary repertoire of sequencing and analytical techniques will be required to detect all BAP1 mutations.

Detection of other driver and putative driver mutations. Of the 139 WES samples analyzed, mutations in Gq pathway genes were detected in 137 (98.6\%) samples and were mutually exclusive in all except two cases (Fig. 1 and Supplementary Fig. 3). SF3B1 mutations were present in $31(22 \%)$ samples and EIF1AX mutations were present in $20(14 \%)$ samples (Supplementary Data 1 and Supplementary Fig. 3). Among samples without a detectable BSE mutation, five were found to harbor mutations in additional spliceosome factors, including RBM10 (two samples), SRSF2 (two samples), and SF3A1 (one sample) (Supplementary Fig. 3). The SRSF2 mutations consisted of deletions encompassing the P95-R102 region previously reported to disrupt splicing in myelodysplastic syndrome ${ }^{21}$. We then reviewed the BSE-mutant samples and found additional mutations in RBM10 (two samples), SRSF2 (one sample), and SRSF7 (one sample). Although the functional consequences of the SF3A1 and SRSF7 mutations are not known, the tumors containing these mutations cluster with the SF3B1-mutant tumors (Fig. 3), suggesting that they may confer similar functional consequences as SF3B1 mutations. Indeed, all of these spliceosome factors have been shown to be mutated in other cancers ${ }^{22-25}$, and they interact functionally with each other and with SF3B1 $1^{26,27}$, which may explain the tendency for these mutations to be mutually exclusive with SF3B1 mutations. Although cancer-associated alterations in SF3B1 are mostly change-of-function hotspot mutations ${ }^{20}$, this is not the case for all splicing factors. For example, RBM10 undergoes frameshifts, truncations, and indels in lung cancer similar to our findings in $\mathrm{UM}^{28}$, suggesting that oncogenic mechanisms may vary among different splicing factor mutations.

Mutational signature. Using a probabilistic modeling algorithm to analyze the 139 WES samples ${ }^{29}$, we identified three mutational signatures (Supplementary Fig. 4). The most prominent signature was a cytosine-to-thymine transition at $\mathrm{CpG}$ dinucleotides, which has been associated with aging ${ }^{30}$. This deamination event accounts for the mutation hotspot at codon 183 in GNAQ and 
GNA11, as well as the mutation hotspot at codon 625 in SF3B1. The second most prominent signature was a cytosine-to-adenine transition, which has been associated with reactive oxygen damage $^{31}$. Interestingly, when samples were analyzed separately based on their BSE mutation, this signature was enriched only in SF3B1 mutant tumors. Thymine-to-guanine transversions were also enriched in all subgroups and are of unknown etiology. Consistent with other reports ${ }^{5,32}$, we found no ultraviolet radiation signature. Notably, despite ostensible links between BAP1 and BRCA1 ${ }^{33}$, no DNA double-strand break-repair "BRCA signature" was detected in BAP1-mutant UMs or any other subgroup, calling into question whether there is in fact a functional link between BAP1 and BRCA1/2 in tumorigenesis ${ }^{33}$.

Methylomic clustering analysis. Previous efforts to classify UMs have been based on CNAs ${ }^{34}$ and $\mathrm{GEP}^{35}$. Here we used global DNA methylation profiling to infer inherent evolutionary relationships between tumors ${ }^{36}$, based on the assumption that uveal melanocytes (from which UMs arise) will have similar methylomic profiles among different individuals. Methylation profiling has the advantage of being agnostic to mutation status and GEP. We used a minimum evolution algorithm ${ }^{37}$ to analyze 87 samples in which global DNA methylation data were available, which revealed phylogenetic clusters that correlate strongly with GEP (class 1 versus class 2) and with BSE mutations (Fig. 3 and Supplementary Fig. 5). Thus, most UMs evolve along one of three canonical trajectories toward fitness maximums denoted by $B A P 1, S F 3 B 1$, or EIF1AX mutations. Most tumors containing alternative spliceosome mutations (RBM10, SRSF2, SF3A1, and $S R S F 7)$ lie between the SF3B1 and BAP1 clusters and may therefore represent intermediate transitional cases or uncommon non-canonical trajectories.

Clonality analysis. Bulk genomic sequencing data provide a chronicle of genomic aberrations that occur during tumor evolution, which can be used to infer the life history of a given tumor $^{38}$. To reveal underlying patterns in the clonal architecture of UM, we analyzed WES data from the 80 TCGA samples and WGS data from the 12 Furney samples ${ }^{20}$ using a hierarchical Bayesian Dirichlet process to identify clonal mutations and the cgpBattenberg algorithm to identify clonal CNAs (Supplementary Fig. 6) ${ }^{38,39}$. As expected, in almost all samples, an initiating Gq pathway mutation was present in $100 \%$ of tumor cells, indicating that it occurred before the appearance of the most recent common ancestor (MRCA) $)^{38}$. Henceforth, we focused on the BSE mutations due to their prognostic gravity, anticipating that they would map to subclones that arose after the MRCA. Surprisingly, however, most samples contained BSE mutations and their associated CNAs (LOH3, 6p+, and $8 \mathrm{q}+$ ) in $100 \%$ of tumor cells (Fig. 4a,b and Supplementary Fig. 7). In a small number of samples, all but one canonical aberration were present in the MRCA, with the other one in a subclone (Fig. 4a,b). For example, five class 1 tumors contained an EIF1AX mutation in the MRCA, with $6 \mathrm{p}+$ arising in a later subclone. Similarly, $\mathrm{LOH} 3$ was always present in $100 \%$ of tumor cells, whereas the associated BAP1 mutations were occasionally found in a subclone. In addition, most alternative spliceosome mutations (RBM10, SRSF2, SF3A1, and SRSF7) mapped to subclones. Hence, the canonical genomic aberrations in UM usually arise in an early punctuated evolutionary process ${ }^{40-42}$, with little ongoing acquisition of new driver aberrations after the appearance of the MRCA.

Evidence for neutral tumor evolution. As our findings suggested that all of the genomic events necessary for the malignant phenotype in UM arise before or soon after the emergence of the
MRCA, we postulated that most or all additional mutations accumulating after the appearance of the canonical genomic aberrations are evolutionarily neutral ${ }^{14,42}$. To test this hypothesis, we used a power-law distribution model to assess the likelihood of neutral tumor growth in WGS data from the 12 Furney samples $^{20}$, as the small number of mutations found in WES data did not allow for proper modeling (Fig. 5a). All 12 samples showed a goodness-of-fit $\left(R^{2}\right)$ well above the 0.98 threshold for neutral tumor evolution (Fig. 5b). Indeed, the UM samples fit the neutral evolution model better than most other cancer types that have been evaluated (Fig. $5 c)^{14}$. These findings support a punctuated evolution model in which the small handful of canonical aberrations necessary to reach a fitness maximum occur early, beyond which there is little ongoing clonal selection or adaptation in the primary tumor.

\section{Discussion}

Historically, cancer has been thought to develop through the gradual accumulation of numerous mutations over long periods of time, with occasional "driver mutations" giving rise to new subclones that evolve under ongoing selective pressure ${ }^{13-15}$. Consistent with recent landmark studies that have challenged this model $^{40-42}$, our findings reveal punctuated rather than gradual evolution in UM. This finding is surprising, as most previous work suggested that CNAs occur in a successive manner in $\mathrm{UM}^{43}$. Most UMs harbor one Gq pathway mutation (GNAQ, GNA11, CYSLTR2, or PLCB4), one BSE mutation (BAP1, SF3B1, or EIF1AX), and a few recurrent CNAs, in $100 \%$ of tumor cells. Hence, these canonical aberrations usually occur relatively early, before the emergence of the MRCA, consistent with punctuated evolution $^{40-42}$. Subsequent aberrations accumulate following a power-law distribution characteristic of neutral tumor evolution $^{14}$. As most UMs cease to accrue subsequent driver mutations once they acquire a BSE mutation, these mutually exclusive aberrations appear to represent alternative fitness maximums, with mutation of one gene relieving selective pressure to mutate the others. Taken together, these findings imply that the metastatic proclivity of a given tumor, which is strongly associated with its respective BSE mutation, may be "set in stone" early in tumor evolution, often before detection of the primary mass, which may explain the lack of improvement in survival rates despite advances in diagnosis and treatment ${ }^{44}$.

Although the canonical aberrations arise through a punctuated evolutionary mechanism, they do not necessarily occur simultaneously. Our findings, taken together with previous work $3,4,7,9,10$ suggest that $\mathrm{Gq}$ pathway mutations are early events required to initiate tumorigenesis but insufficient alone for malignant transformation, whereas BSE mutations confer malignant potential but are unable to trigger clonal expansion without a $\mathrm{Gq}$ mutation. Thus, BSE mutations either arise subsequent to Gq mutations or, if they occur first, they remain silent unless "unleashed" by a Gq mutation. The latter mechanism is presumably operative in patients with a germline BAP1 mutation, two of which were included in this study. In both cases, a $\mathrm{Gq}$ mutation was present in $100 \%$ of tumor cells but was absent in the germline. Hence, a preexistent $B A P 1$ mutation was present in all uveal melanocytes but clonal expansion only occurred after one of these cells acquired a Gq mutation. This need for a tumor-initiating $\mathrm{Gq}$ mutation could explain why only a minority of individuals with germline BAP1 mutations develop $\mathrm{UM}^{45}$. It is also interesting that several samples analyzed for clonality harbored more than one BSE mutation. AA9A, A985 and T11 contained BAP1 and SF3B1 mutations, whereas A9EC contained SF3B1 and EIF1AX mutations. As all of these BSE mutations were present in the MRCA, they most likely coexisted in the same tumor cells rather than in 
a

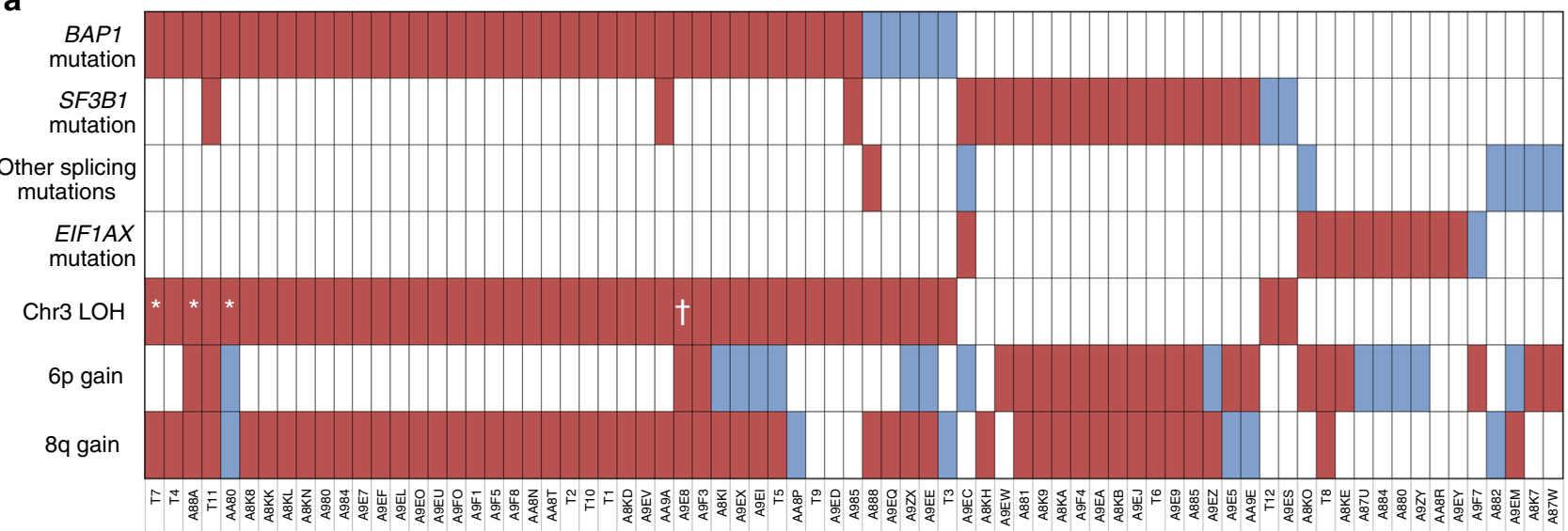

Present in $\sim 100 \%$ of tumor cells (emerged before most recent common ancestor)

Present in a subclone of tumor cells (emerged after most recent common ancestor)

b

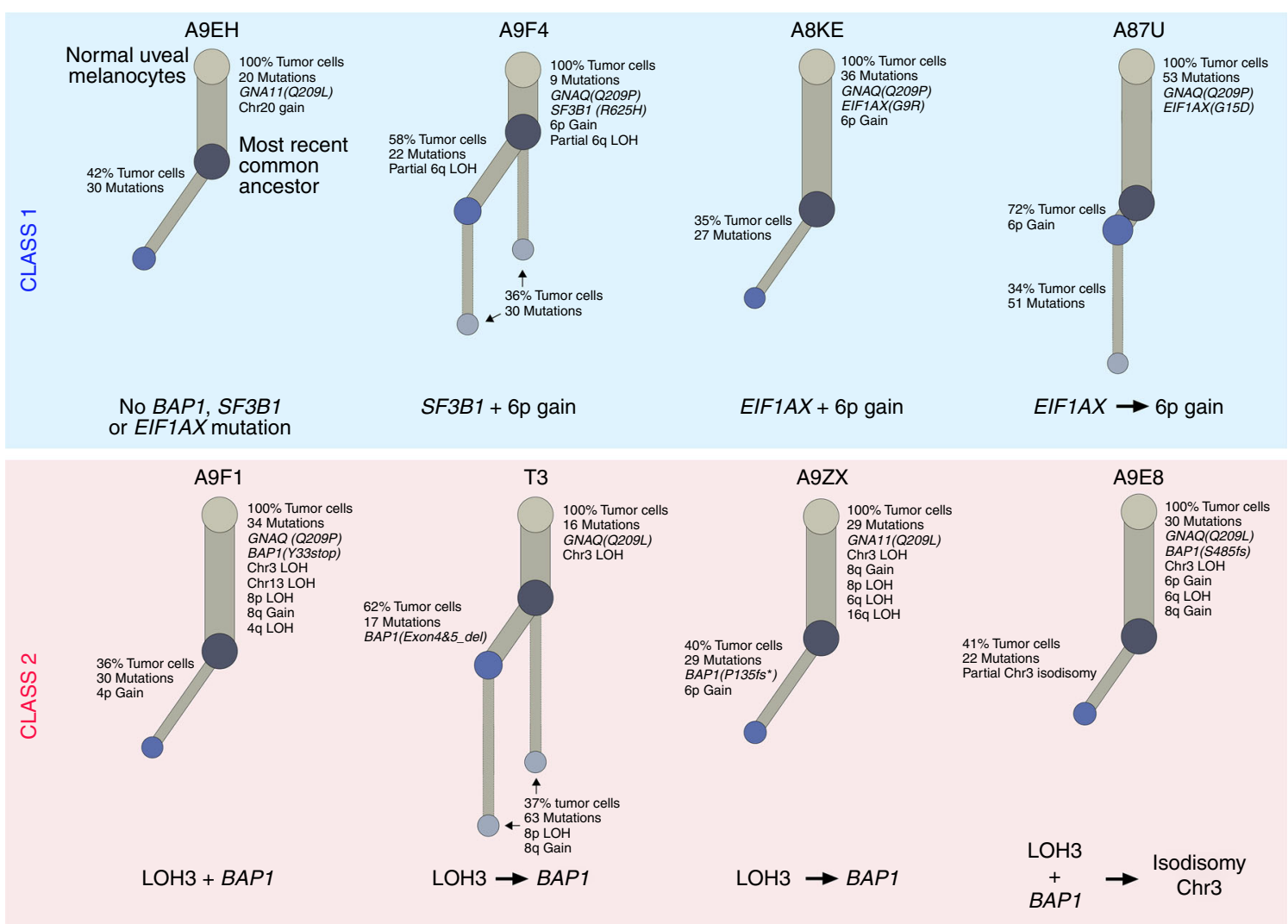

Fig. 4 Clonal evolution in uveal melanoma. a Clonal relationships between canonical driver mutations and chromosome copy number alterations. Red bars denote aberrations that are present in $100 \%$ of tumor cells and thus arising before the most recent common ancestor (MRCA). Blue bars denote aberrations present in a subclone of $<100 \%$ tumor cells arising subsequent to the MRCA. A white bar denotes that the aberration was not present. ${ }^{\star}$ Isodisomy 3 in $100 \%$ of tumor cells; fisodisomy 3 in a subclone of $<100 \%$ of tumor cells. $\mathbf{b}$ Life history clonal evolution plots for eight representative uveal melanoma samples. Blue box illustrates class 1 tumors with no BSE mutations (A9EH), SF3B1 and 6p gain in 100\% of tumor cells (A9F4), EIF1AX and $6 \mathrm{p}$ gain in $100 \%$ of tumor cells (A8KE), and EIF1AX in 100\% of tumor cells and 6p gain in a subclone (A87U). Red box illustrates class 2 tumors with LOH3 and BAP1 in 100\% of tumor cells (A9F1), LOH3 in 100\% of tumor cells, and BAP1 mutation in a subclone (T3 and A9ZX), and LOH3 and BAP1 in 100\% of tumor cells with later duplication of chromosome 3 to generate isodisomy 3 in a subclone (A9E8). The node at the top of each plot (light grey circle) represents normal uveal melanocytes, the presumed precursor cell that gives rise to all uveal melanomas. The next lower node (dark gray circle) represents the most recent common ancestor (MRCA) from which all aberrations present in 100\% of tumor cells arose. Lower nodes (blue circles) represent subclones that arise after the most MRCA, with the area of the node being proportional to the percentage of cells in the subclone. The mutations and CNAs that occur between two nodes are indicated beside the connecting branch (gray bar). The length of each branch is proportional to the number of mutations that occur between nodes and the width of the branch is proportional to the percentage of cells containing those mutations. When a node contains rare aberrations that cannot be accurately mapped to one node, it is mapped to all possible nodes, as described in Methods 
a
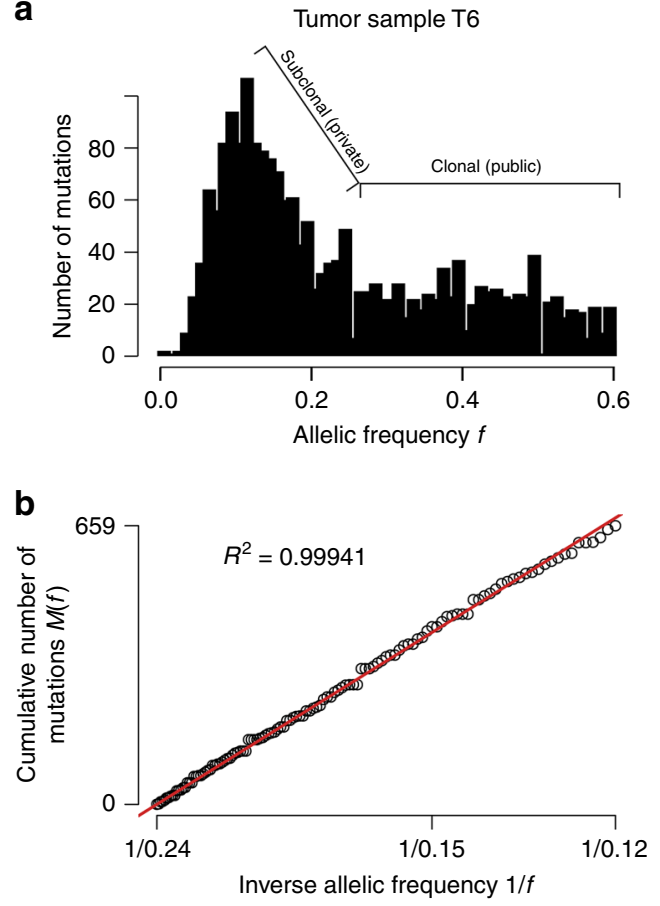

C

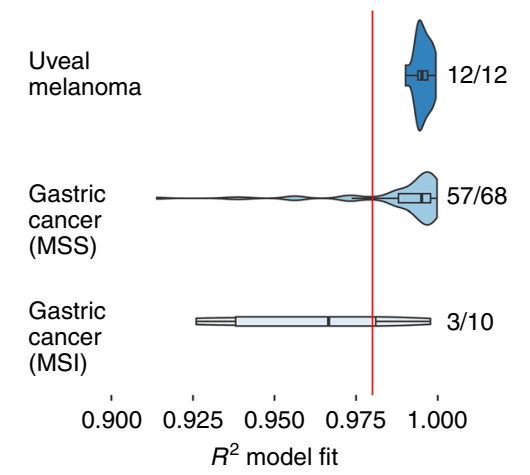

Fig. 5 Analysis of uveal melanoma whole genome sequencing (WGS) data for evidence of neutral tumor evolution. a Histogram of mutant allele frequency for representative tumor sample T6. Mutations that occur at frequencies $\geq 0.25$ are considered to be clonal (public) and those occurring at frequencies below this cutoff are subclonal (private) ${ }^{14}$. b Cumulative distribution, $M(f)$, of subclonal mutations for sample $T 6$, which is highly consistent with the neutral evolution mode ${ }^{14}$. c Comparison of goodnessof-fit, $R^{2}$, for neutral tumor evolution in uveal melanoma versus previously published gastric cancer WGS data ${ }^{14}$. All 12 uveal melanoma samples demonstrated $R^{2} \geq 0.98$ (red line), whereas 60 of 78 (76.9\%) gastric cancer samples without microsatellite instability (MSS) and only $3 / 10$ (30\%) of gastric cancer samples with microsatellite instability (MSS) met this threshold. The proportion of samples with a particular $R^{2}$ model fit is proportional to the violin plot width. Box plots within the violin plot illustrate the median, upper, and lower quartiles, and Tukey's whiskers (median \pm 1.58 times interquartile range)

different tumor subclones. Interestingly, the GEP in these cases reflected the mutation associated with the worse prognosis.

Although most class 2 tumors contained a BAP1 mutation and LOH3 in the MRCA, the BAP1 mutation (but not LOH3) was occasionally present in a subclone, suggesting that $\mathrm{LOH} 3$ can precede mutation of $B A P 1$ on the other chromosome 3. $6 \mathrm{p}+\mathrm{Was}$ usually present in $100 \%$ of tumor cells in SF3B1-mutant tumors but only in a subclone in EIF1AX-mutant and BAP1-mutant tumors; $8 \mathrm{q}+$ was usually present in $100 \%$ of tumor cells in SF3B1- mutant and $B A P 1$-mutant tumors but was rarely present in EIF1AX tumors. 8q+ In class 2/BAP1-mutant tumors usually consisted of multiple extra copies of the entire $\mathrm{q}$ arm, whereas in class 1/SF3B1-mutant tumors, it usually involved simple gain of a smaller fragment of 8q. There was no "smallest common region" of amplification to implicate a specific gene(s) under selective pressure. Taken together, CNAs appear to evolve along the evolutionary trajectory of their associated BSE mutation. Future studies are warranted to further delineate the early events in UM evolution in greater detail.

Of all the canonical aberrations in UM, the single most prognostically significant is the bi-allelic inactivation of $B A P 1$, which is tightly linked to the class 2 GEP prognostic signature and high metastatic risk ${ }^{9}$. As such, we developed an enhanced bioinformatic pipeline to discover additional BAP1 mutations. We show here that optimal detection of $B A P 1$ mutations requires a variety of different bioinformatic techniques, including special realignment of reads around large indels, use of multiple mutation callers, detection of homozygous deletions, and "SpliceDel" analysis of RNA-Seq and WGS data, to detect deletions spanning multiple exons or encompassing untranscribed regions. These findings indicate that the frequency of BAP1 mutations in UM and other cancer types may be considerably underestimated. As expected, we did not observe an increased frequency of detecting other canonical mutations since they are mostly SNPs that are easily detected.

This study discloses a wide variety of previously unreported BAP1 and spliceosome mutations, and identifies punctuated evolution as an organizing principle behind the genetic, genomic, and transcriptomic landscape of UM. The early emergence of prognostically significant BSE mutations could explain why micrometastasis frequently occurs before diagnosis ${ }^{46}$, and why more aggressive primary tumor treatment has not resulted in improved survival ${ }^{44}$. Consequently, these findings support an intensified effort to develop more effective treatments for metastatic disease.

\section{Methods}

Patients and sample collection. Tumor and blood samples from the JWH data set were obtained from patients in the practice of the senior author who were diagnosed with UM arising from the choroid and/or ciliary body, and treated by primary enucleation without previous radiotherapy. The study was approved by the Institutional Review Board at the University of Miami and written informed consent was obtained from each patient. Clinical and histopathologic information were obtained and de-identified for further analyses. WES was performed on 40 tumor samples, 21 of which had matched blood DNA samples available for sequencing. DNA was extracted using the Wizard Genomic DNA Purification kit (Promega, Madison, WI) and the Quick Gene DNA whole blood kit S (Fugifilm, Tokyo, Japan), respectively. Exome fragments were captured using NimbleGen SeqCap EZ Human Exome Library v2.0 (Roche Nimblegen) and sequenced on the Illumina Genome Analyzer II (GAIIx). RNA from these cases was isolated using the PicoPure kit and sent to Castle BioSciences, Inc. for GEP to determine class 1 versus class 2 status ${ }^{47}$.

NGS data sources, quality control, and alignment. Raw data files from 80 TCGA UM samples was obtained from the Cancer Genomics Hub (CGHub). Raw data files from the Martin et al. ${ }^{11}$ and Furney et al. ${ }^{20}$ raw data were obtained from the European Genome-phenome Archive (EGA). Data sets provided in BAM format (TCGA; Furney et al. ${ }^{20}$ ) were converted back into FASTQ files using bamUtil: bam2FastQ (v1.0.13) and were adjusted into proper format with unique read names using CGAT (v0.2.4 $)^{48}$. FASTQ files from all sources, including our data, then underwent the following bioinformatics pipeline. Sequence data were quality controlled using FastQC (v0.11.3). WES and WGS reads were trimmed (if required) and aligned to the human genome (hg19/GRCH37) using Novoalign (v3.04.06), marked for duplicates using Picard (v1.128), realigned around small and large indels using ABRA (v0.94c) ${ }^{18}$, and read mate fixed and analyzed for coverage statistics using Picard. Tumor samples that had $<30 \mathrm{x}$ coverage were excluded from further analysis. Unknown or unplaced contigs and mitochondrial genes were excluded from analysis. Raw RNA-seq FASTQ files from TCGA were obtained from CGHub and from Furney et al. ${ }^{20}$ via EGA, assessed for quality using FastQC, and aligned to the genome using STAR (v2.5) ${ }^{49}$. For research purposes, GEP class 
status of TCGA samples was estimated using the following method ${ }^{16}$. RNA-seq normalized count files were generated using DESeq2 in $\mathrm{R}^{50}$. Unsupervised principal component analysis (PCA) was conducted on the top $20 \%$ most variable expressed genes and plotted three-dimensionally using the stats and rgl packages in $\mathrm{R}$, which grouped the samples into two clusters, as has been previously described for Class 1 and Class 2 tumors ${ }^{47}$. The identity of each cluster was determined to be most consistent with Class 1 versus Class 2 based on the expression of genes previously known to be differentially upregulated in each Class. Class assignment showed $100 \%$ concordance with the 11 TCGA samples in which Class status was determined using the clinically available DecisionDx-UM test. This method was used solely for research purposes and is not meant for actual clinical testing, as it has not been prospectively validated in a manner analogous to the DecisionDx-UM test.

NGS mutation calling. WES data sets underwent variant calling for SNPs and Indels using Varscan2 (v2.4.1) ${ }^{51}$ and MuTect2 (GATK 2016-01-25 nightly build) ${ }^{52}$. Combining the two mutation callers was based on the finding that Varscan2 detects more true somatic and high-confidence SNPs than MuTect, whereas MuTect is better at detecting low-coverage SNPs. ${ }^{53}$ MuTect2 is an updated SNP and indel mutation caller available from GATK that combines the original MuTect with the assembly-based machinery of HaplotypeCaller. MuTect2 has its own incorporated local realignment that adjusts for tumors with purity less than $100 \%$, multiple subclones, and/or copy number variation (either local or aneuploidy). Varscan2 output was further refined using bam-readcount and a false positive filter (fplfilter) 54 with filter parameters set based on the TCGA-ICGC DREAM-3 SNV Challenge results, with adjustment of minimum average read trim length. For MuTect2, mutation calls for a panel of normals ( $n=117$ germline blood samples) was generated and pooled together to further filter out mutations that were present in at least two normals. For tumors without matched blood samples, MuTect2 was used for variant calling with a panel of normals and a high coverage blood sample to filter out likely germline mutations. MuTect2 was utilized for mutation calls of the WGS samples. To assess whether any mutations were missed by our pipeline due to low coverage, tumor-blood matched WES data were analyzed in combination with matched RNA-Seq data from the tumor using UNCeqR (v.0.2) ${ }^{19}$ with default settings. In addition to the default settings for UNCeqR, mutations were filtered if both DNA and RNA alternate tumor read counts were $<3,<20 \%$ of the total tumor read count, or the gene fell within the blacklist suggested by Radia ${ }^{55}$. For mutations called by Varscan2 or MuTect2, mutations were further filtered out if both alternate tumor read counts were $<3$ and the minor allele frequency (MAF) was $<20 \%$. For all sequencing samples, the BAM files were investigated manually for the regions of interest (BAP1, SF3B1, EIF1AX, GNAQ, CYSLTR2, PLCB4, and GNA11) using the IGV (v2.3.80, Broad Institute, Cambridge, MA). STAR-aligned RNA-seq data from the TCGA samples was also evaluated for "alternative splicing" within BAP1 exons, indicative of deletions not detected in the corresponding WES data due to the design baits for exome capture. The same strategy was used on the Furney et al..$^{20}$ WGS data set in which all regions of the genome are covered.

For all called mutations, annovar was used for annotation ${ }^{56}$. Following annotation, mutations were filtered out if the MAF was $1 \%$ or greater in the population according to 1000 Genomes Project (2015 August) or Exome Sequencing Project (2015 March), and mutations listed in dbSNP (v138) were filtered out, with the exception of SNPs with a MAF $<1 \%$ (or unknown) in the population, a single mapping to reference assembly, or with a "clinically associated" tag. Functional consequences of SNPs were assessed by three mutational predictor tools: Polyphen2 (probably damaging, possibly damaging, and benign) ${ }^{57}$, FATHMM (damaging and tolerated) ${ }^{58}$, and MetaLR (damaging and tolerated) ${ }^{59}$ In non-exonic regions and for synonymous mutations, SNPs were considered deleterious if two out of three of the above prediction algorithms predicted a damaging mutation. For non-synonymous exonic SNPs, mutations were not considered deleterious if two out of three algorithms predicted a benign or tolerated mutation. Probably damaging, possibly damaging, or damaging were considered deleterious calls. All insertions and deletions in exonic regions and alterations in splicing junctions were considered deleterious. A summary of the driver mutations, GEP status (class 1 versus class 2), source of tumor sample, and availability of matched normal DNA were mapped in a co-occurrence of mutations plot using ComplexHeatma ${ }^{60}$. Lollipop plots displaying the distribution of driver mutations along the protein domains of each gene was plotted using

MutationMapper (http://www.cbioportal.org/mutation_mapper.jsp) and domain information was populated based on default annotations in combination with a literature review ${ }^{61}$

After mutation calling, mutational signature analysis and visualization was conducted on WES data using the pmsignature package in R, which infers mutational signatures based on probabilistic models, similar to mixed-membership models used in population genetics and machine learning ${ }^{29}$. Signatures were modeled for nucleotide base changes with different combinations of \pm 2 flanking bases using default parameters, which included a maximum of 10,000 iterations, a minimum of 10 iterations with different starting values, and a tolerance of estimation of 1e-04. Different numbers of mutational signatures were modeled, and the number of signatures was selected where the overall log-likelihood was highest without a rise in bootstrap error and where any given inferred signature did not show a similar pattern across the genome to another inferred member. This analysis was conducted on all samples in combination, as well as on samples broken down into BSE subgroups.

Copy number aberrations. Copy number gains and losses were determined from WES and WGS data using CNVKit (v0.7.5, v0.7.10.dev0) ${ }^{62}$ and by cgpBattenberg ${ }^{38}$ for clonality analysis using default settings. Raw SNP 6.0 arrays from the TCGA data set and raw HumanOmni2.5 SNP arrays from the Furney et al. ${ }^{20}$ data set were analyzed using ASCAT(v2.4 ${ }^{63}$ with default settings. Isodisomy was determined by plotting MAF plots using CNVKit, ASCAT, TITAN ${ }^{64}$, and cgpBattenberg.

\section{Phylogenetic analysis using DNA methylation data. Raw output from the} Infinium HumanMethylation450 BeadChip Kit (Illumina) was downloaded from TCGA $(n=80)$, and combined with our own cases $(n=7)$ and analyzed using the ChAMP R package ${ }^{65}$. The top $20 \%$ most variable methylation probe $\beta$-values were used for unsupervised PCA analysis using the stats and rgl R packages. Phylogenetic evolutionary models were generated using the minimum evolution algorithm (Canberra distance) in the ape R package ${ }^{66}$. Likelihoods of each bipartition in the tree were calculated using bootstrapping with 100 replicates.

Clonality analysis. To determine the clonality of copy number gains and losses, we used cgpBattenberg, which determines chromosome copy number gains and losses, and corrects for cell contamination of normal cells ${ }^{38,67}$. Adaptation of cgpBattenberg scripts and dependencies was performed by the High Performance Computing Core, Center for Computational Science at the University of Miami, for proper installation on the CentOS operating system used by the Pegasus supercomputer. Clusters of mutations were determined using a Bayesian Dirichlet process model that involves Gibbs sampling to estimate the posterior distribution of the parameters of interest. Mutations were assigned to the cluster in which they were most likely to fall based on variant allele frequency, following adjustment for normal cell contamination and copy number gains and losses using previously described algorithms and publicly available $\mathrm{R}$ scripts $^{38}$. This methodology minimizes the risk of underestimating indel frequencies relative to SNPs by assuming that all mutations in the first cluster are in the MRCA. Two independent researchers reviewed the mutation cluster plots and counted the number of discrete clusters. When disagreement existed, the higher cluster number was selected. Subclones in which the mean of the cluster had a tumor read count $<10 \%$ of the allelic fraction were excluded as an independent subclone due to the possibility that these mutations could be spread across multiple different clones. Mutations attributed to this subclone were assigned to the next smallest cluster. For each cluster, the 95\% confidence interval fitted for the Bayesian Dirichlet process model was used to calculate the number of mutations in the cluster. Subclonal copy number gains and losses, mutation clusters, and adjusted mutation variant allele frequency information were integrated using the pigeonhole principle to determine phylogenetic trees for each sample ${ }^{38,67}$. A custom python script was developed to automate generation of intra-tumor evolutionary trees, which takes inputs from the above analyses and generates an editable scalable vector graphics (.svg) file visualizing the life history, or phylogenetic tree, for a given sample. This algorithm can be provided upon request. Combining cgpBattenberg and the Bayesian Dirichlet process model for clonality analysis of CNAs and mutations, respectively, is the only publicly available methodology at this time for simultaneously determining the clonal evolution of CNAs and mutations occurring in the same genomic region. Other clonality algorithms are limited in that they either (1) only evaluate mutations in regions without CNAs or (2) pair mutations with CNAs in regions with CNAs and assume they occur together.

Neutral tumor evolution. To assess whether the evolution of UM fits a Darwinian versus a neutral tumor evolution model, we analyzed the 12 Furney et al. ${ }^{20}$ WGS samples using a simple power-law distribution model predicted by neutral growth $^{14}$. Mutations with variant allele frequencies between $12 \%$ and $24 \%$ were selected to account only for reliably called subclonal mutations and tumor purity in the samples. Proper modeling required samples to have a minimum of 12 mutations within this fitness boundary ${ }^{14}$. WES samples were not used for this analysis because none of them met this criterion. A custom script was provided by Williams et al. ${ }^{14}$. A goodness-of-fit $\left(R^{2}\right)$ value of greater than 0.98 was considered to positively fit a neutral tumor evolution model. Calculated $R^{2}$ values for UM WGS cases were compared to $R^{2}$ values from WGS gastric cancer cases and plotted in a violin plot using ggplot2 in $\mathrm{R}$.

Data availability. All sequencing and methylation array data generated from the practice of the senior author (J.W.H.) have been deposited in and are available from the dbGaP database under dbGaP accession phs001421.v1.p1.

Received: 20 March 2017 Accepted: 29 November 2017 Published online: 09 January 2018 


\section{References}

1. Onken, M. D. et al. Collaborative Ocular Oncology Group report number 1: prospective validation of a multi-gene prognostic assay in uveal melanoma. Ophthalmology 119, 1596-1603 (2012).

2. Decatur, C. L. et al. Driver mutations in uveal melanoma: associations with gene expression profile and patient outcomes. JAMA Ophthalmol. 134, 728-733 (2016).

3. Van Raamsdonk, C. D. et al. Frequent somatic mutations of GNAQ in uveal melanoma and blue naevi. Nature 457, 599-602 (2009).

4. Van Raamsdonk, C. D. et al. Mutations in GNA11 in uveal melanoma. N. Engl. J. Med. 363, 2191-2199 (2010).

5. Johansson, P. et al. Deep sequencing of uveal melanoma identifies a recurrent mutation in PLCB4. Oncotarget 7, 4624-4631 (2015).

6. Moore, A. R. et al. Recurrent activating mutations of G-protein-coupled receptor CYSLTR2 in uveal melanoma. Nat. Genet. 48, 675-680 (2016).

7. Onken, M. D. et al. Oncogenic mutations in GNAQ occur early in uveal melanoma. Invest. Ophthalmol. Vis. Sci. 49, 5230-5234 (2008).

8. Bauer, J. et al. Oncogenic GNAQ mutations are not correlated with disease-free survival in uveal melanoma. Br. J. Cancer 101, 813-815 (2009).

9. Harbour, J. W. et al. Frequent mutation of BAP1 in metastasizing uveal melanomas. Science 330, 1410-1413 (2010).

10. Harbour, J. W. et al. Recurrent mutations at codon 625 of the splicing factor SF3B1 in uveal melanoma. Nat. Genet. 45, 133-135 (2013).

11. Martin, M. et al. Exome sequencing identifies recurrent somatic mutations in EIF1AX and SF3B1 in uveal melanoma with disomy 3. Nat. Genet. 45, 933-936 (2013).

12. Harbour, J. W. The genetics of uveal melanoma: an emerging framework for targeted therapy. Pigment. Cell. Melanoma Res. 25, 171-181 (2012).

13. Vogelstein, B. et al. Cancer genome landscapes. Science 339, 1546-1558 (2013).

14. Williams, M. J., Werner, B., Barnes, C. P., Graham, T. A. \& Sottoriva, A Identification of neutral tumor evolution across cancer types. Nat. Genet. 48, 238-244 (2016).

15. Shain, A. H. et al. The genetic evolution of melanoma from precursor lesions. N. Engl. J. Med. 373, 1926-1936 (2015).

16. Field, M. G. et al. Epigenetic reprogramming and aberrant expression of PRAME are associated with increased metastatic risk in Class 1 and Class 2 uveal melanomas. Oncotarget 7, 59209-59219 (2016).

17. Broad Institute TCGA Genome Data Analysis Center (2016): Analysis Overview for Uveal Melanoma (Primary solid tumor cohort) - 28 January 2016. Broad Institute of MIT and Harvard.

18. Mose, L. E., Wilkerson, M. D., Hayes, D. N., Perou, C. M. \& Parker, J. S. ABRA: improved coding indel detection via assembly-based realignment. Bioinformatics 30, 2813-2815 (2014)

19. Wilkerson, M. D. et al. Integrated RNA and DNA sequencing improves mutation detection in low purity tumors. Nucleic Acids Res. 42, e107 (2014).

20. Furney, S. J. et al. SF3B1 mutations are associated with alternative splicing in uveal melanoma. . Cancer Discov. 3, 1122-1129 (2013).

21. Komeno, Y. et al. SRSF2 is essential for hematopoiesis, and its myelodysplastic syndrome-related mutations dysregulate alternative pre-mRNA splicing. Mol. Cell. Biol. 35, 3071-3082 (2015).

22. Yoshida, K. et al. Frequent pathway mutations of splicing machinery in myelodysplasia. Nature 478, 64-69 (2011).

23. Witkiewicz, A. K. et al. Whole-exome sequencing of pancreatic cancer defines genetic diversity and therapeutic targets. Nat. Commun. 6, 6744 (2015).

24. Imielinski, M. et al. Mapping the hallmarks of lung adenocarcinoma with massively parallel sequencing. Cell 150, 1107-1120 (2012).

25. Sanborn, J. Z. et al. Phylogenetic analyses of melanoma reveal complex patterns of metastatic dissemination. Proc. Natl Acad. Sci. USA 112, 10995-11000 (2015).

26. Matera, A. G. \& Wang, Z. A day in the life of the spliceosome. Nat. Rev. Mol. Cell. Biol. 15, 108-121 (2014)

27. Hegele, A. et al. Dynamic protein-protein interaction wiring of the human spliceosome. Mol. Cell. 45, 567-580 (2012).

28. Zhao, J. et al. Functional analysis reveals that RBM10 mutations contribute to lung adenocarcinoma pathogenesis by deregulating splicing. Sci. Rep. 7, 40488 (2017).

29. Shiraishi, Y., Tremmel, G., Miyano, S. \& Stephens, M. A simple model-based approach to inferring and visualizing cancer mutation signatures. PLoS Genet. 11, e1005657 (2015).

30. Alexandrov, L. B. et al. Signatures of mutational processes in human cancer. Nature 500, 415-421 (2013).

31. Kino, K. \& Sugiyama, H. Possible cause of G-C $\rightarrow$ C-G transversion mutation by guanine oxidation product, imidazolone. Chem. Biol. 8, 369-378 (2001)

32. Royer-Bertrand, B. et al. Comprehensive genetic landscape of uveal melanoma by whole-genome sequencing. Am. J. Hum. Genet. 99, 1190-1198 (2016).

33. Jensen, D. E. et al. BAP1: a novel ubiquitin hydrolase which binds to the BRCA1 RING finger and enhances BRCA1-mediated cell growth suppression. Oncogene 16, 1097-1112 (1998).
34. Trolet, J. et al. Genomic profiling and identification of high-risk uveal melanoma by array CGH analysis of primary tumors and liver metastases. Invest. Ophthalmol. Vis. Sci. 50, 2572-2580 (2009).

35. Onken, M. D., Worley, L. A., Ehlers, J. P. \& Harbour, J. W. Gene expression profiling in uveal melanoma reveals two molecular classes and predicts metastatic death. Cancer Res. 64, 7205-7209 (2004).

36. Capra, J. A. \& Kostka, D. Modeling DNA methylation dynamics with approaches from phylogenetics. Bioinformatics 30, i408-i414 (2014).

37. Desper, R. \& Gascuel, O. Fast and accurate phylogeny reconstruction algorithms based on the minimum-evolution principle. J. Comput. Biol. 9 687-705 (2002).

38. Nik-Zainal, S. et al. The life history of 21 breast cancers. Cell 149, 994-1007 (2012).

39. Cooper, C. S. et al. Analysis of the genetic phylogeny of multifocal prostate cancer identifies multiple independent clonal expansions in neoplastic and morphologically normal prostate tissue. Nat. Genet. 47, 367-372 (2015).

40. Navin, N. et al. Tumour evolution inferred by single-cell sequencing. Nature 472, 90-94 (2011).

41. Sottoriva, A. et al. A Big Bang model of human colorectal tumor growth. Nat Genet. 47, 209-216 (2015).

42. Gao, R. et al. Punctuated copy number evolution and clonal stasis in triplenegative breast cancer. Nat. Genet. 48, 1119-1130 (2016).

43. Hoglund, M. et al. Dissecting karyotypic patterns in malignant melanomas: temporal clustering of losses and gains in melanoma karyotypic evolution. Int J. Cancer 108, 57-65 (2004).

44. Singh, A. D. \& Topham, A. Survival rates with uveal melanoma in the United States: 1973-1997. Ophthalmology 110, 962-965 (2003).

45. Wiesner, T. et al. Germline mutations in BAP1 predispose to melanocytic tumors. Nat. Genet. 43, 1018-1021 (2011).

46. Eskelin, S., Pyrhonen, S., Summanen, P., Hahka-Kemppinen, M. \& Kivela, T. Tumor doubling times in metastatic malignant melanoma of the uvea - tumor progression before and after treatment. Ophthalmology 107, 1443-1449 (2000)

47. Harbour, J. W. \& Chen, R. The DecisionDx-UM gene expression profile test provides risk stratification and individualized patient care in uveal melanoma. PLoS Curr. https://doi.org/10.1371/currents.eogt.af8ba80fc776c8f1ce8f5dc48 $5 \mathrm{~d} 4 \mathrm{a} 618$ (2013)

48. Sims, D. et al. CGAT: computational genomics analysis toolkit. Bioinformatics 30, 1290-1291 (2014).

49. Dobin, A. et al. STAR: ultrafast universal RNA-seq aligner. Bioinformatics 29 , 15-21 (2013)

50. Anders, S. et al. Count-based differential expression analysis of RNA sequencing data using R and Bioconductor. Nat. Protoc. 8, 1765-1786 (2013).

51. Koboldt, D. C. et al. VarScan 2: somatic mutation and copy number alteration discovery in cancer by exome sequencing. Genome Res. 22, 568-576 (2012).

52. Cibulskis, K. et al. Sensitive detection of somatic point mutations in impure and heterogeneous cancer samples. Nat. Biotechnol. 31, 213-219 (2013).

53. Xu, H., DiCarlo, J., Satya, R. V., Peng, Q. \& Wang, Y. Comparison of somatic mutation calling methods in amplicon and whole exome sequence data. $B M C$ Genomics 15, 244 (2014).

54. Koboldt, D. C., Larson, D. E. \& Wilson, R. K. Using VarScan 2 for germline variant calling and somatic mutation detection. Curr. Protoc. Bioinformatics $\mathbf{4 4}$ 15.4.1-17 (2013).

55. Radenbaugh, A. J. et al. RADIA: RNA and DNA integrated analysis for somatic mutation detection. PLoS ONE 9, e111516 (2014).

56. Wang, K., Li, M. \& Hakonarson, H. ANNOVAR: functional annotation of genetic variants from high-throughput sequencing data. Nucleic Acids Res. 38, e164 (2010).

57. Adzhubei, I. A. et al. A method and server for predicting damaging missense mutations. Nat. Methods 7, 248-249 (2010).

58. Shihab, H. A., Gough, J., Cooper, D. N., Day, I. N. \& Gaunt, T. R. Predicting the functional consequences of cancer-associated amino acid substitutions. Bioinformatics 29, 1504-1510 (2013).

59. Dong, C. et al. Comparison and integration of deleteriousness prediction methods for nonsynonymous SNVs in whole exome sequencing studies. Hum Mol. Genet. 24, 2125-2137 (2015).

60. Gu, Z., Eils, R. \& Schlesner, M. Complex heatmaps reveal patterns and correlations in multidimensional genomic data. Bioinformatics 32, 2847-2849 (2016).

61. Harbour, J. W. \& Chao, D. L. A molecular revolution in uveal melanoma: implications for patient care and targeted therapy. Ophthalmology 121, 1281-1288 (2014)

62. Talevich, E., Shain, A. H., Botton, T. \& Bastian, B. C. CNVkit: genome-wide copy number detection and visualization from targeted DNA sequencing. PLoS Comput. Biol. 12, e1004873 (2016).

63. Van Loo, P. et al. Allele-specific copy number analysis of tumors. Proc. Natl Acad. Sci. USA 107, 16910-16915 (2010)

64. Ha, G. et al. TITAN: inference of copy number architectures in clonal cell populations from tumor whole-genome sequence data. Genome Res. 24, 1881-1893 (2014). 
65. Morris, T. J. et al. ChAMP: 450k chip analysis methylation pipeline. Bioinformatics 30, 428-430 (2014).

66. Paradis, E., Claude, J. \& Strimmer, K. APE: analyses of phylogenetics and evolution in R language. Bioinformatics 20, 289-290 (2004).

67. Gundem, G. et al. The evolutionary history of lethal metastatic prostate cancer. Nature 520, 353-357 (2015).

\section{Acknowledgements}

We are grateful to the patients who generously contributed samples for this research. We thank Lisle Mose and Eric Talevich for their assistance with the ABRA and CNVkit programs, respectively. We acknowledge the support of the Biostatistics \& Bioinformatics and Oncogenomics Shared Resources at the Sylvester Comprehensive Cancer Center, and the University of Miami Center for Computational Science. The data used here were generated in part by the TCGA Research Network, Cancer Research UK Manchester Institute, Institut Curie, and University of Duisburg-Essen. This work was supported by National Cancer Institute grants R01 CA125970 (J.W.H.), R01 CA161870 (J.W.H. and A. M.B.) and F30 CA206430 (M.G.F.), Research to Prevent Blindness, Inc. Senior Scientific Investigator Award (J.W.H.), Melanoma Research Foundation (J.W.H. and M.G.F.), Melanoma Research Alliance (J.W.H.), Ocular Melanoma Foundation (J.W.H.), the 2015 RRF/Kayser Global Pan-American Award (J.W.H.), the Alcon Research Institute (J.W. H.), the Sylvester Comprehensive Cancer Center (J.W.H.), the University of Miami Sheila and David Fuente Graduate Program in Cancer Biology (M.G.F. and M.A.D.), the Center for Computational Science Fellowship (M.G.F. and M.A.D.), the AACR-Ocular Melanoma Foundation Fellowship in honor of Robert C. Allen, MD (S.K.), and a generous gift from Dr. Mark J. Daily (J.W.H.). The Bascom Palmer Eye Institute also received funding from NIH Core Grant P30EY014801, Department of Defense Grant \#W81XWH-13-10048, and a Research to Prevent Blindness Unrestricted Grant.

\section{Author contributions}

M.G.F. analyzed and interpreted the data, and wrote the manuscript. M.A.D. analyzed and interpreted the data, and edited the manuscript. H.A. and L.C. analyzed the data. C. D. collected and prepared tissue samples. A.B. interpreted the data. S.K. wrote the script to plot evolutionary trees, interpreted the data, and edited the manuscript. J.W.H designed and led the project, provided clinical samples, interpreted the data, and wrote the manuscript.

\section{Additional information}

Supplementary Information accompanies this paper at https://doi.org/10.1038/s41467017-02428-w.

Competing interests: Drs. Harbour and Bowcock are inventors of intellectual property discussed in this study. Dr. Harbour is a paid consultant for Castle Biosciences, licensee of this intellectual property, and he receives royalties from its commercialization. All remaining authors declare no competing financial interests.

Reprints and permission information is available online at http://npg.nature.com/ reprintsandpermissions/

Publisher's note: Springer Nature remains neutral with regard to jurisdictional claims in published maps and institutional affiliations.

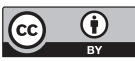

Open Access This article is licensed under a Creative Commons Attribution 4.0 International License, which permits use, sharing, adaptation, distribution and reproduction in any medium or format, as long as you give appropriate credit to the original author(s) and the source, provide a link to the Creative Commons license, and indicate if changes were made. The images or other third party material in this article are included in the article's Creative Commons license, unless indicated otherwise in a credit line to the material. If material is not included in the article's Creative Commons license and your intended use is not permitted by statutory regulation or exceeds the permitted use, you will need to obtain permission directly from the copyright holder. To view a copy of this license, visit http://creativecommons.org/ licenses/by/4.0/.

(C) The Author(s) 2018 